\title{
Lipochimie et innovations
}

Oléagineux, Corps Gras, Lipides. Volume 10, Numéro 5-6, 344-7, Double 5-6, SEPTEMBRE-OCTOBRENOVEMBRE-DÉCEMBRE 2003, INNOVATION, RÉGLEMENTATION, ÉCOCOMPATIBILITÉ

Auteur(s) : Carine ALFOS, Institut des Corps Gras, ITERG, Rue Monge, 33600 Pessac, France $<$ c.alfositerg.com>.

Author(s) : Carine ALFOS

Résumé : Peut-on parler d'un nouvel âge d'or pour les produits à base de ressources végétales renouvelables et notamment pour les huiles? Porteurs de performances spécifiques et de caractéristiques plus écologiquement correctes, leur emploi ouvre à l'industrie un potentiel d'innovations auquel participe le département de chimie et rhéologie de l'Iterg. La prise de conscience collective, associée aux évolutions des réglementations françaises et européennes, devrait conduire les industries à prendre de plus en plus en compte le respect de l'environnement dans leurs activités. La substitution de dérivés pétrochimiques par des produits d'origine agricole dans certains secteurs industriels (détergence, lubrifiants, solvants notamment) contribue à la protection de notre environnement: diminution des volumes de déchets et des effluents, lutte contre l'effet de serre, moindre écotoxicité, innocuité pour la santé. De plus, l'épuisement latent des ressources pétrolières et la pratique de la jachère ont permis d'accélérer le développement de nouvelles voies de valorisations des huiles végétales et plus largement des agroressources. Les enjeux, à l'horizon 2010, sont bien plus importants encore pour ces produits, qui de part leur fonctionnalité spécifique, ont vocation à continuer d'occuper des marchés de niche. D'ici 10 ans, 2 à 3 millions d'hectares pourraient être consacrés à l'industrie du végétal $\backslash$; il s'agit là d'une véritable stratégie de développement durable qui nécessite un soutien politique, une vision à long terme et une motivation forte de l'industrie.

Summary : Can we speak of a new gold age for products from renewable vegetable resources and especially for vegetable oils ? With specific performances and more environmental characteristics, their use leads to a great innovation potential for industrials $\backslash$; the chemical and rheological department of Iterg works on that way to promote their uses. The collectivity awareness joined by the french and european regulations evolutions, should lead industries to take more and more into account, environmental respect in their activities. The substitution of petrochemical derivatives by agricultural origin products in some industrial sectors (mainly detergency, lubricants and solvents) promotes the respect of environment: loss decreasing, greenhouse effect fightening and less eco toxicity. Moreover, using up of petrochemical resources and the fallow field practice, have promoted to accelerate the development of new vegetable oils valorizations fields (and agricultural resources in general). The perspectives in 2010 are much more important for these products whom specific functionality's will lead them to niche market occupancy. In 2010, 2 to 3 millions ha could be devoted to vegetable industry $\backslash$; it is a real strategy of durable development which need a political support, a long term view and a strong industry motivation. 
Mots-clés : oléochimie, biolubrifiants, biosolvants

Keywords : oleochemistry, biolubricant, biosolvent

ARTICLE

Auteur(s) : Carine ALFOS

Institut des Corps Gras, ITERG, Rue Monge, 33600 Pessac, France

<c.alfos@iterg.com>

\section{Données économiques}

La production mondiale des huiles végétales et des graisses est passée en vingt ans (1963/1984) d'un peu plus de $30 \mathrm{Mt}$ à $66 \mathrm{Mt}$. En 1997, elle a dépassé les $100 \mathrm{Mt}$ dont $80 \mathrm{Mt}$ d'origine végétale et $20 \mathrm{Mt}$ d'origine animale. Plus de $80 \%$ de ces huiles et graisses sont utilisées en alimentation humaine. Le reste se répartit entre l'industrie de l'alimentation animale [5] et les besoins de l'oléochimie [15] soit pour cette dernière, une consommation de 15 à $17 \mathrm{Mt}$ d'huiles végétales, l'huile de palme et l'huile de soja demeurant et de loin, les matières premières les plus utilisées [1]. Le tableau 1 présente les estimations de consommation des bases oléochimiques les plus couramment utilisées sur la période 2000 à 2010 et leur poids relatif par rapport à la consommation mondiale.

Tableau 1. Estimation pour 2000, 2005 et 2010 de la consommation des bases oléochimiques par zone géographique et par famille chimique (millions tonnes) [2].

\begin{tabular}{|l|l|l|l|}
\hline & 2000 & 2005 & 2010 \\
\hline Total mondial & 5,76 & 6,69 & 7,75 \\
\hline Europe de l'ouest & $1,76(31 \%)$ & $1,87(28 \%)$ & $1,96(25 \%)$ \\
\hline Amérique du Nord & $1,36(24 \%)$ & $1,52(23 \%)$ & $1,66(21 \%)$ \\
\hline Asie & $2,27(39 \%)$ & $2,79(42 \%)$ & $3,54(46 \%)$ \\
\hline Autres & $0,37(6 \%)$ & $0,51(7 \%)$ & $0,59(8 \%)$ \\
\hline Acides gras & 3,05 & 3,50 & 4,00 \\
\hline Esters méthyliques & 0,66 & 0,73 & 0,80 \\
\hline Alcools & 1,44 & 1,73 & 2,07 \\
\hline Aminés & 0,57 & 0,62 & 0,70 \\
\hline Glycérol & 0,75 & 0,86 & 1,00 \\
\hline
\end{tabular}




\section{Évolutions réglementaires}

L'impact environnemental des économies industrielles et de certains produits, et la prise de conscience des phénomènes associés, ont conduit notamment les pays industrialisés à élaborer en 1997 un protocole international d'accord, dit de Kyoto, les engageant à respecter des quotas de réduction de leurs émissions de gaz à effet de serre (GES) pour la période 2008-2012 à hauteur de $5,2 \%$ sur la base des émissions de 1990. Au niveau européen, la directive 99/13/CE encadre désormais les installations utilisatrices de solvants, élargissant notamment l'emploi de produits à basse teneur en solvant dans le secteur des peintures et des vernis. Le projet finalisé en vue de sa transposition en droit français devrait voir le jour au cours du premier trimestre 2004.

\section{Atout des huiles végétales et de leurs dérivés}

Matières premières plutôt plus élaborées que les bases équivalentes de la pétrochimie, les huiles végétales avec leur composition en acides gras définie, bien que variable, et leur groupements carboxyliques présentent des caractéristiques physicochimiques remarquables (pour obtenir un type de produit équivalent à partir de bases pétrochimiques, l'oligomérisation de l'éthylène, puis un traitement selon le procédé " oxo » conduisant à l'obtention d'un mélange statistique de composés carboxyliques est nécessaire). Leurs propriétés anti-friction naturelles élevées, leur polarité due à leur structure moléculaire amphiphile les rendent en particulier directement utilisables, ou presque, dans différents secteurs tels que celui des lubrifiants, des solvants et des agents de surface.

\section{Deux secteurs de valorisation des huiles végétales : lubrifiants et solvants}

Suite au rapport de Philippe Desmarescaux [16] sur "les stratégies de développement des productions agricoles non alimentaires " (décembre 1998), cinq axes prioritaires concernant la filière oléagineuse ont été identifiés ${ }^{1}$ : biolubrifiants, tensioactifs et autres produits détergents, solvants et intermédiaires chimiques, acides aminés et biocarburants. Ces secteurs se développent aujourd'hui significativement en Europe et de nombreux produits performants sont présents sur les marchés, en particulier dans les domaines des biolubrifiants et des biosolvants.

${ }^{1}$ Ces axes ont été retenus sur la base de critères de sélection portant sur la rentabilité économique, l'importance des surfaces agricoles concernées, et le caractère positif des bilans énergétiques et environnementaux.

\section{Les lubrifiants}

Les lubrifiants sont fabriqués à partir d'huiles d'origine minérale ou végétale, additivées à hauteur d'au moins $3 \%$ d'additifs. Les huiles végétales, après transformation chimique, peuvent être employées soit comme huiles de base soit comme additifs. Parmi les $40 \mathrm{Mt}$ de lubrifiants consommés au niveau mondial-États-Unis $13 \mathrm{Mt}$, Europe 10,1 Mt et Asie 13,2 Mt-seulement $1 \mathrm{Mt}$ d'huiles végétales est utilisé. Le marché reste donc ouvert étant donné le contexte réglementaire rappelé précédemment.

À niveau de performances identiques, les aspects de biodégradabilité deviennent déterminants. Ces éléments favorisent le développement des huiles végétales seules ou en mélange (mixte végétal/minéral).

Au niveau européen, la consommation de lubrifiants a représenté en 2000 un total de 5 millions de tonnes dont $50 \%$ pour le secteur automobile et $35 \%$ pour l'industrie (métallurgie, huiles 
hydrauliques, fluides caloporteurs). Pour des raisons d'ordre économique (disponibilité, rapport performances/prix intéressants pour les applications actuelles), $90 \%$ du marché est dominé par des lubrifiants sur base minérale, mélange d'un grand nombre de molécules de type paraffines, naphténiques et aromatiques composés peu favorables au respect de l'environnement [4]. À ce jour, plus de la moitié des quantités utilisées n'est pas récupéré et, de ce fait, constitue une source de pollution préoccupante (épandage accidentel ou non, combustion sauvage, lessivage par les eaux de pluie, évaporation). Au niveau français, la consommation des lubrifiants s'est élevée en 2001 à environ 860000 tonnes réparties entre les lubrifiants automobiles (455 000 tonnes), les lubrifiants pour les industries (295000 tonnes) et les huiles de procédés (109000 tonnes). Parmi les huiles multigrades, les qualités les plus fluides représentent près de $96 \%$. Pour répondre à une demande croissante et pour des raisons d'économie de consommation, les formulateurs orientent leurs choix vers des bases synthétiques peu visqueuses et présentant une certaine onctuosité tels que les esters (éventuellement combinés avec des modificateurs de frottement) permettant de garantir un critère dit de "Fuel economy" tout au long de la durée d'utilisation du lubrifiant [5]. La part du marché européen des lubrifiants d'origine végétale avoisine $2 \%$, soit 100000 tonnes, et inclut principalement les formules à partir d'huiles de tournesol et de colza [6]. Dans de nombreux secteurs d'activité à travers toute l'Europe se sont multipliés des exemples réussis d'utilisation de biolubrifiants d'origine végétale :

- pour l'exploitation forestière, les pays Scandinaves utilisent déjà largement des bases végétales pour les huiles de scie et pour les huiles hydrauliques,

- pour le bâtiment et les travaux publics, les huiles de décoffrage habituellement utilisées sont des bases minérales diluées dans du kérosène ; en France, le programme " Chantiers verts " [7] a conduit des entreprises à privilégier les huiles de décoffrage à base végétale,

- pour le transport ferroviaire français, les esters biodégradables sont en cours d'homologation par la SNCF.

Parmi les différentes bases utilisées dans les formulations précitées, les esters de polyols d'acides gras sont les plus courants et possèdent les propriétés recherchées en lubrification, à savoir :

- un bon comportement viscosité - température,

- un bas point d'écoulement,

- une faible volatilité,

- un bon pouvoir lubrifiant,

- une stabilité élevée à l'oxydation thermique.

Les tableaux 2 et 3 établissent une comparaison entre les propriétés physiques et les performances de divers lubrifiants sur base minérale ou végétale ainsi que les avantages liés à l'utilisation d'une huile lubrifiante à base d'esters d'acides gras. 
Tableau 2. Comparaison entre les propriétés physiques et les performances de divers lubrifiants sur base minérale ou végétale.

\begin{tabular}{|l|l|l|l|l|l|l|}
\hline $\begin{array}{l}\text { Type de } \\
\text { lubrifiant }\end{array}$ & $\begin{array}{l}\text { Indice de } \\
\text { viscosité } \\
\text { température à basse }\end{array}$ & $\begin{array}{l}\text { Propriétés } \\
\text { lubrifiantes }\end{array}$ & $\begin{array}{l}\text { Stabilité thermique } \\
\text { et à l'oxydation }\end{array}$ & $\begin{array}{l}\text { Basse } \\
\text { volatilité }\end{array}$ \\
\hline Huiles minérales & F-M & F-M & F-M & F-M & M-B \\
\hline $\begin{array}{l}\text { Monoesters } \\
\text { d'acides gras }\end{array}$ & B-E & F & B-E & M & B-B \\
\hline $\begin{array}{l}\text { Diesters d'acides } \\
\text { gras }\end{array}$ & B-E & E & B & B & & B-E \\
\hline Esters de polyols & M-E & M-E & B-E & & \\
\hline
\end{tabular}

$\mathrm{F}$ : faible ; $\mathrm{M}$ : moyen ; $\mathrm{B}$ : bon ; $\mathrm{E}$ : excellent

Tableau 3. Avantages liés à l'utilisation d'une huile lubrifiante à base d'esters d'acides gras [8].

\begin{tabular}{|c|c|c|}
\hline Propriétés & Résultats & Bénéfices \\
\hline Anti-usure & Usure réduite & $\begin{array}{l}\text { Faible consommation d'huile } \\
\text { Longévité accrue du matériel }\end{array}$ \\
\hline Onctuosité & $\begin{array}{l}\text { Bonne adhérence du produit sur } \\
\text { le } \quad \text { support } \\
\text { Persistance d'un film lipophile }\end{array}$ & $\begin{array}{l}\text { Protection contre la corrosion } \\
\text { Réduction des frottements mécaniques } \\
\text { Economie de consommation des carburants } \\
\text { ("Fuel economy") }\end{array}$ \\
\hline $\begin{array}{l}\text { Bonne solubilité des } \\
\text { additifs }\end{array}$ & Performances accrues & Compatibilité avec nouveaux additifs \\
\hline $\begin{array}{l}\text { Pouvoir dispersant } \\
\text { Stabilité thermique } \\
\text { Antioxydant }\end{array}$ & Propreté du mécanisme & $\begin{array}{l}\text { Additivation } \\
\text { Intervalle de véduite } \\
\text { Maintenance réduite }\end{array}$ \\
\hline Eco-compatibilité & $\begin{array}{l}\text { Biodégradabilité } \\
\text { Moindre toxicité }\end{array}$ & $\begin{array}{llr}\text { Meilleure } & \text { image } \\
\text { Respect de l'environnement } & \\
\text { Réduction des taxes } & \end{array}$ \\
\hline
\end{tabular}


Pour faciliter l'émergence du marché des lubrifiants biodégradables des mesures incitatives et normatives ont été prises par d'autres pays européens, en particulier l'Allemagne : obligation pour certains marchés (huiles perdues) ou préconisation pour d'autres (industries alimentaires). II semble donc nécessaire d'instaurer dans ce domaine une cohérence européenne. Un travail important reste à faire compte tenu du très faible développement de ces produits en France. Les derniers travaux de l'Iterg dans ce domaine ont porté sur la fabrication de lubrifiants utilisés dans le démoulage du béton (voir encadré).

\section{Lubrifiants pour le décoffrage et le démoulage du béton [9]}

Les huiles de démoulage sont assimilées à des travaux d'une grande banalité consistant à interposer entre une surface d'acier ou de bois (le moule) et du béton un produit destiné à créer une perte d'adhérence et rendre la séparation des deux entités. Les huiles végétales constituent d'excellentes bases pour la formulation des produits de démoulage ; quelques formulations sont présentes sur le marché : pures et en coupe avec des huiles minérales et plus ou moins diluées avec des solvants hydrocarbonés afin de répondre aux aspects techniques de pulvérisation sur chantier. La mise en émulsion aqueuse à l'aide d'émulgateurs est un excellent moyen de formuler des produits de démoulage permettant de régler le couple viscosité/extrait sec nécessaire à la pulvérisation et permettant d'ajuster l'épaisseur de l'agent de démoulage déposé. La modification chimique des huiles végétales a permis d'obtenir de très bonnes compatibilités de ces dérivés avec l'eau allant de la solubilité (microémulsions) à l'auto émulsification. Les émulsions ainsi formulées, pulvérisées entre le moule et le béton permettent d'obtenir un bon parement du béton, après son démoulage, et également de ne pas polluer le moule après utilisation par des résidus de produit sec ou des salissures. Ces travaux ont été réalisés dans le cadre d'un projet AGRICE en partenariat avec la société Chryso et Vandeputte.

\section{Les solvants}

En Europe, $23 \%$ des composés organiques volatils (COV) rejetés dans l'atmosphère, provient des solvants [3]. Du fait de leur polarité et sans composés aromatiques, les solvants d'origine végétale, tels que les esters méthyliques, trouvent une place de plus en plus importante en tant qu'agent de substitution aux solvants d'origine pétrochimique. L'industrie des peintures et des vernis utilise, en formulation, des quantités très importantes de solvants organiques et volatils (plus de 5 millions de tonnes de solvants par an en Europe). Il s'agit le plus souvent de solvants hydrocarbonés. Les récentes directives européennes visent à diminuer de plus de $57 \%$ les émissions de solvants organiques volatils (COV). II faut donc diminuer de $320 \mathrm{~g}$ de solvants/litre pour les plus grandes tolérances à $5 \mathrm{~g} /$ litre voire 0 pour les secteurs les plus sévères [10]. Le développement des peintures aqueuses est loin d'être suffisant pour diminuer les émissions de COV car elles ne peuvent être utilisées dans toutes les applications. La France produit un peu plus de 800000 tonnes de peintures et de vernis par an. L'utilisation des solvants représente plus de 200000 tonnes. II faut donc à terme atteindre une réduction de 
100000 tonnes dans l'hypothèse la plus défavorable d'une formule à $320 \mathrm{~g} /$ litre maximum. L'étude et l'utilisation des esters méthyliques d'huiles végétales ont fait l'objet d'un programme européen de recherche [11] montrant toutes les qualités environnementales ainsi que d'hygiène et de sécurité des esters méthyliques d'acides gras à longues chaînes; les applications étudiées et rapportées [12] concernent les solvants lourds dans le dégraissage et le nettoyage de métaux avant mise en peinture ou encore les encres d'imprimerie. United Soyabean Board reprend ce thème en l'étendant aux formulations touchant la cosmétique, les produits de nettoyage ménagers et industriels, les peintures, les produits anti graffitis et les décapants. Des travaux réalisés sur des solvants réactifs du type adduits maléiques estérifiés par de l'alcool allylique [13] montrent que des doubles liaisons peuvent réagir et participer à la formation du film de peinture tout en apportant un pouvoir solvant à la formulation et à l'application. Un exemple industriel réussi concerne l'utilisation des biosolvants dans la formulation des produits phytosanitaires [14]. En effet, si les traitements phytosanitaires sont indispensables à la conduite des cultures, deux constats s'imposent: d'une part on estime qu'entre 1 et $10 \%$ (seulement) de la matière active atteint sa cible biochimique et, d'autre part, l'utilisation de produits phytosanitaires est désignée comme une des sources majeures de pollution des nappes d'eau. Les solvants sont utilisés dans les concentrés émulsionnables où ils permettent la solubilisation des matières actives. Les solvants traditionnellement utilisés étaient d'origine pétrolière, tels que xylène, naphta et les solvants aromatiques lourds. Les huiles végétales et leurs esters de monoalcools, s'ils sont correctement formulés, sont de réelles alternatives aux solvants d'origine minérale. Ces produits améliorent l'efficacité de la matière active et son taux d'utilisation, tout en assurant la sécurité de I'utilisateur et le respect de l'environnement. L'Iterg continue à promouvoir l'utilisation des esters méthyliques d'huiles végétales (EMHV) en remplacement de ces solvants d'origine pétrochimique, dans différentes applications. Ainsi, des formulations à base d'EMHV ont été testées dans des formulations de vernis à ongles. Le temps de séchage du vernis ainsi que le "rendu couleur » ont été appréciés comparativement aux formules classiques et ont mis en évidence des performances intéressantes pour ces produits. Dans un tout autre secteur d'application, le développement de I'utilisation des EMHV en tant que " liant " dans les formulations de bitume en lieu et place des fractions légères d'hydrocarbures aromatiques représente aujourd'hui des tonnages conséquents [15].

\section{Conclusion}

L'utilisation de matières premières renouvelables d'origine agricole pour la fabrication de bioproduits dans le domaine de la chimie correspond à une double nécessité : d'une part, offrir une alternative aux ressources fossiles et d'autre part, répondre aux défis environnementaux actuels : lutte contre l'effet de serre, réduction des pollutions (air, sol, eau), innocuité et biodégradabilité des produits. La biomasse peut assurer une véritable alternative comme matière première de la pétrochimie compte tenu du risque connu de pénurie des ressources fossiles et donc de leur renchérissement, face à une demande croissante d'énergie. La compétitivité des matières premières végétales et la taille des marchés varient dans de très grandes proportions de la niche au secteur potentiellement énorme des biocarburants. Les matières premières renouvelables ne peuvent répondre à toutes les attentes des secteurs potentiellement utilisateurs, mais leur bilan positif mérite un soutien politique, une vision à long terme et une motivation de l'industrie. Si l'importance du renouvelable fait aujourd'hui l'objet d'une prise de conscience et d'un intérêt croissant au niveau européen, les produits verts ne s'imposeront vraiment que si ils sont compétitifs et de qualité. 
Des efforts de recherche importants sont consacrés à la création de cette nouvelle chimie ; ces efforts doivent être accrus et continus. Ils impliquent une coordination active entre des acteurs aussi variés que les équipes de recherche pluridisciplinaires, les entreprises agro-industrielles et les entreprises des secteurs utilisateurs ainsi que les filières agricoles. La nécessité de cette coordination se trouve encore renforcée, aujourd'hui compte tenu du rôle clé de ces filières " non alimentaires " dans le domaine de l'environnement. C'est dans cet esprit que les pouvoirs publics et huit partenaires ont créé en 1994 le groupement d'intérêt scientifique AGRICE : Agriculture pour la Chimie et l'Energie qui soutient des programmes de recherche visant à substituer des produits issus du végétal aux produits d'origine fossile [17]. AGRICE a su structurer la recherche sur ce marché naissant, constituer une plate forme du bio renouvelable en France et susciter des applications concrètes; le nouveau groupement a été renouvelé pour une nouvelle période de 6 ans à compter de 2001 avec des orientations qui tiennent compte de l'expérience et des évolutions de contexte.

\section{RÉFÉRENCES}

1. Acides et alcools gras : une source irremplaçable de produits chimiques, Info Chimie Magazine, $n^{\circ} 417$, Avril 2000.

2. GUNSTONE FD. Basic oleochemicals products and new industrial oils, Oleochemical Manuf. Appl., p. 1-22, 2001.

3. CLAUDE S. Oleochemistry as an opportunity to consolidate the sustainable development of European oil crops, an updateed prospect, OCL, Vol. 6, n 5, septembre/octobre 1999.

4. Lubrifiants 2001, Statistiques, Centre Professionnel des Lubrifiants.

5. Industries et Techniques, $n^{\circ}$ 782, Mai 1997, p. 161-163.

6. Lubricants based on vegetable oils, Petroleum and coal, vol. 41, 2, 1999.

7. Lubrifiants d'origine naturelle : quel avenir pour le développement de cette filière ? L'actualité chimique, octobre 1999.

8. Les lubrifiants à base d'esters de néopentylpolyols, OCL Vol. 3, n 1, janvier/février 1996.

9. Formulation d'agents de démoulage solubles ou auto émulsionnables en milieu aqueux à base de dérivés d’huiles végétales, Agrice $n^{\circ} 0101$ 038, 2001.

10. Usine nouvelle, hors série 2000.

11. VOFApro AIR-CT94-2063.

12. NIELSEN C, WALLSTROM E. Fäarg och Lack Scandinavia 5/98.

13. Nremberg Congress, april 1999.

14. CHARLEMAGNE D. Apport de la lipochimie à l'industrie phytosanitaire, $O C L$ Vol. $6, n^{\circ} 5$, septembre/octobre 1999. 
15. ALFOS C, DAVID K, MARCKLOUX J. Formulation de fluctuants pour bitumes à base d'esters méthyliques d’huiles végétales. $O C L$, Vol. 10, n5 5-6, septembre-décembre 2003.

16. DEMARESCAUX P. Place de la lipochimie dans le développement des productions agricoles à usage non alimentaire, OCL, Vol. 6, n 5, septembre/octobre 1999.

17. Bilan d'activité AGRICE 1994-2000. 